\title{
THE INFLUENCE OF THE SURGICAL TECHNIQUE USED FOR VASECTOMY ON TESTIS FUNCTION IN RATS
}

\author{
G. V. HELLER AND I. ROTHCHILD \\ Department of Reproductive Biology, \\ Case Western Reserve University School of Medicine, \\ Cleveland, Ohio 44106, U.S.A.
}

(Received 9th October 1973)

Recent studies have suggested that vasectomy or vasoligation can have deleterious effects on testis structure and function in rats. Sackler, Weltman, Pandhi \& Schwartz (1973) found a decrease in the testis weights and total 17-ketosteroid excretion 28 weeks after bilateral vasectomy or vasoligation of immature rats performed without aseptic precautions; the decrease in 17ketosteroid excretion was observed as early as 15 weeks after the operation. Thakur, Sheth \& Rao (1972) reported decreased testis weights, but increased androgenic activity (as judged by prostatic maltase activity) in unilaterally and bilaterally vasoligated adult rats, using a 'semi-sterile' technique. These results are in conflict with earlier studies (Moore \& Quick, 1924; Smith, 1962) in which only transient increases of testis weights were found after vasectomy.

It is possible that decreased testicular activity could be the result of the conditions under which the ductus is ligated or removed. To test this, vasectomies were performed under either relatively sterile or non-sterile conditions and the results were compared.

Fifty-one adult male Holtzman- (Sprague-Dawley) strain rats weighing 350 to $400 \mathrm{~g}$ were housed under a $14-\mathrm{hr}$ light $/ 10$-hr dark schedule at $76^{\circ} \mathrm{F}$, and were allowed free access to Purina Rat Chow. In rats subjected to 'sterile' vasectomy, the scrotum and abdomen were shaved, cleaned with soap and water, soaked liberally with $70 \%$ ethanol, and draped with sterile towels. All instruments and ligatures were kept in $70 \%$ ethanol when not in use, and for at least $30 \mathrm{~min}$ before use. After completion of the operation, the area was again cleaned with $70 \%$ ethanol, but was not dressed. In the 'non-sterile' technique, the area was shaved and clean instruments were used, but the area was not washed, soaked with ethanol, or draped, and the instruments were not kept in ethanol. With both techniques, either a scrotal or mid-line abdominal incision was used to expose the ductus deferens. Two silk ligatures (Ethicon 0-0) were placed approximately $1 \mathrm{~cm}$ apart around the ductus and a cut was made between them.

The primary comparison was between the 'sterile' and 'non-sterile' techniques, but comparisons were also made between the abdominal and scrotal approaches, with either technique. Within the group in which the 'sterile' technique was used, a comparison was also made between ligation and non- 


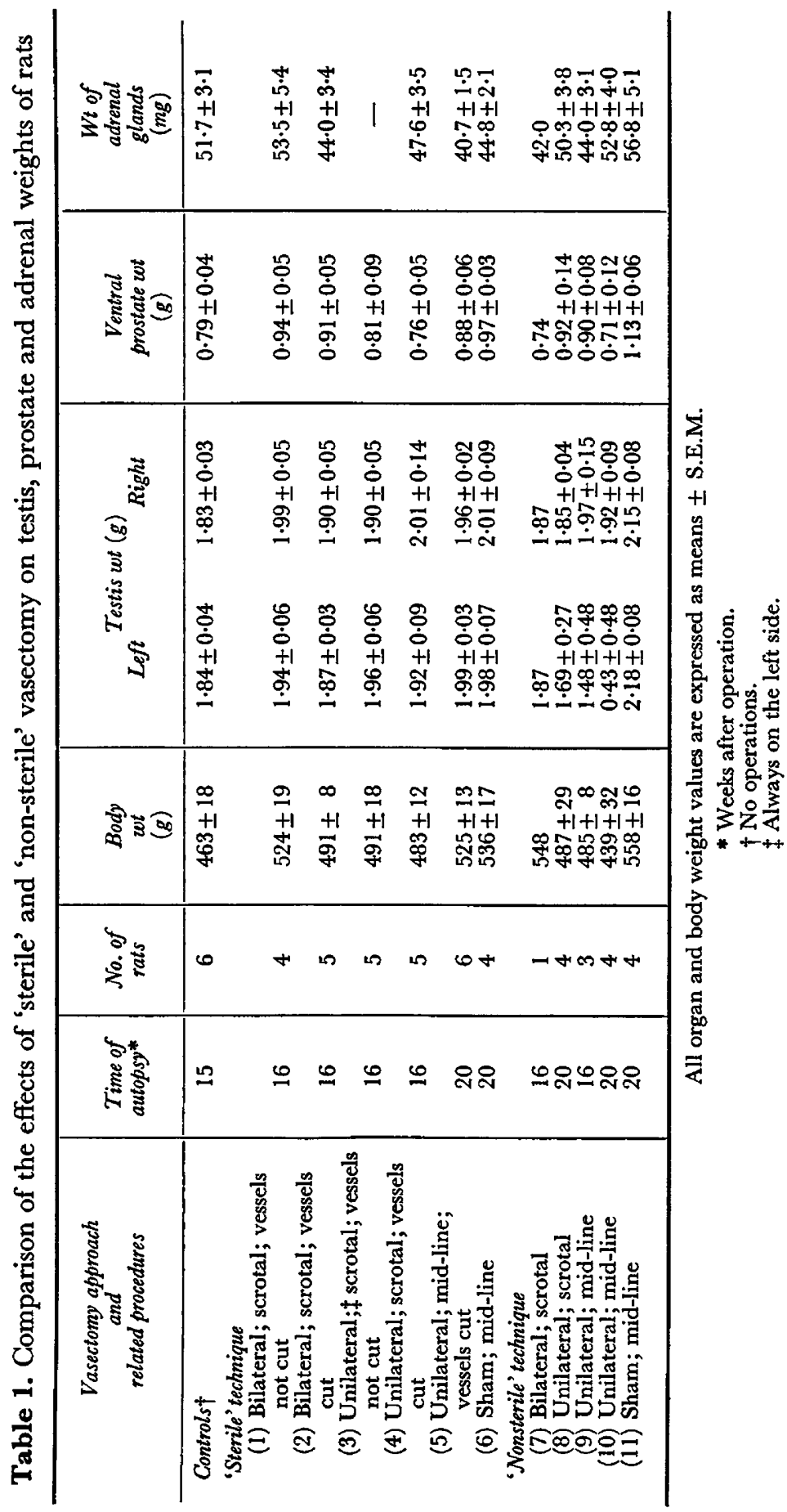


ligation of the deferential blood vessels. In several groups, the effect of a given combination of procedures was observed by performing them on the left side only, the right testis serving as a control.

The rats were killed either 16 or 20 weeks after operation. The testes, adrenals, ventral prostate, seminal vesicles and coagulating glands were weighed, and the operation site was inspected for indications of infection or other effects of the surgery. The data were statistically analysed by one-way analysis of variance for unequal replicates, or by Student's $t$ test.

In $95 \%$ of the 'sterile' and 'non-sterile' vasectomized rats, one or more discrete yellow masses, 0.4 to $3.0 \mathrm{~cm}$ in length, were found near the distal end of the ductus deferens. On microscopic examination, they were found to be spermatocoeles, containing densely packed degenerated spermatozoa. Spermatocoeles have also been described by other investigators, including Smith (1962) and Sackler et al. (1973), in association with vasectomy.

Within the 'sterile' group, there were no significant differences from the controls (sham or no operation) in the body, testis, prostate or adrenal weights, regardless of the site of the incision, ligation of blood vessels, or whether the vasectomy was unilateral or bilateral (Table 1). There was also no significant difference between the seminal vesicle or coagulating gland weights of the 'sterile' and 'non-sterile' groups, or within each group. None of the twenty-five vasectomized rats in the 'sterile' group showed any significant evidence of infection. By contrast, five of the eleven rats unilaterally vasectomized by the 'non-sterile' technique (in Groups 8, 9, 10, Table 1) had purulent abscesses in the area of the ligatures and numerous adhesions to the surrounding tissues. The testes on the ligated side in two of these animals were markedly reduced in weight $(0.9 \mathrm{~g}$ and $0.5 \mathrm{~g})$ and could not be identified in the remaining three. The contralateral testes in these rats were normal in weight and appearance. Four of the five infected rats had been vasectomized by the mid-line abdominal approach.

Analysis of variance indicated that, as far as the testis, ventral prostate and body weights were concerned, the rats in the 'sterile' group were a homogenous population. This was not true for those in the 'non-sterile' group, probably because of the difference between Group 10 (Table 1) and the others. When the left testis weights in the 'non-sterile' group were compared with left testis weights in the 'sterile' group (by Student's $t$ test), the mean of the former was significantly smaller $(P<0.0005)$ than that of the latter.

These results demonstrate that vasectomy performed under relatively sterile surgical conditions had no adverse effect on the testis or the male accessory organs. Vasectomy performed without any precautions to prevent infection, however, caused severe losses in testis weight in some animals. Our data suggest, therefore, that the alterations in testis structure and function described by Sackler et al. (1973) and Thakur et al. (1972) were primarily related to the complications of the surgical procedure rather than to a specific effect of vasectomy or vasoligation itself. The increased white blood cell counts in their experimental groups (when compared with the control animals) would tend to support this conclusion.

Our findings support those of Plaut (1973); he found little or no change in 
testis size or function after vasectomy of rats treated post-surgically with antibiotics. Plaut found, however, that ligation of the deferential blood vessels, with bilateral vasectomy, caused a slight but significant decrease in the left but not in the right testis weight, in comparison with intact controls. He noted that adhesions, when present, were found only on the left side. This again suggests that the alterations observed were not due specifically to the vasectomy.

Sackler et al. (1973) used their findings to question the practice of human vasectomy as a means of contraception. Our data indicate that one must be cautious in comparing species, and especially so when the effects of a surgical procedure are involved.

This research was aided in part by NIH-NICHD Training Grant HD-00024 and Ford Foundation Training Grant 67-135. One of us (G.V.H.) was a Predoctoral Trainee in Reproductive Biology. We wish to thank Miss Helen Wilk for her assistance with the experiments.

\section{REFERENCES}

MOORE, G. R. \& Quick, W. J. (1924) Properties of the gonads as controllers of somatic and psychical characteristics. VII. Vasectomy in the rabbit. Am. F. Anat. 34, 317.

Plaut, S. M. (1973) Testicular morphology in rats vasectomized as adults. Science, N.Y. 181, 554.

Sackler, A. M., Weltman, A. S., Pandhi, V. \& Schwartz, R. (1973) Gonadal effects of vasectomy and vasoligation. Science, N.Y. 179, 293.

SMITH, G. (1962) The effects of ligation of the vasa efferentia and vasectomy on testicular function in the adult rat. 7. Endocr. 23, 385.

Thakur, A. N., Sheth, A. R. \& RAo, S. S. (1972) Biochemical studies on rat testes and sex accessory organs after vasoligation operation. Fert. Steril. 23, 834. 